\title{
Independent Registration and Virtual Controlled Reduction of Pelvic Ring Fractures
}

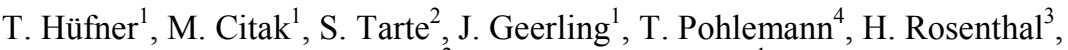 \\ L.P. Nolte ${ }^{2}$ and C. Krettek, M.D. ${ }^{1}$ \\ ${ }^{1}$ Trauma Department, Hannover Medical School, 30625 Hannover, Germany \\ Hüfner.Tobias@mh-hannover.de \\ ${ }^{2}$ Department for Orthopedic Biomechanics, M. E. Müller Institute, Bern, Switzerland \\ ${ }^{3}$ Radiology Dept., Hannover Medical School, Hannover, Germany \\ ${ }^{4}$ Trauma Department, University of Saarland, 66421 Homburg, Germany
}

\begin{abstract}
This study presents a new developed software module for Computer Assisted Surgery (Surgigate, Medivision, Oberdorf, Switzerland), allowing independent registration of two fragments and real time virtual representation while reduction occurs.

Three fracture models were used to evaluate the accuracy: geometric foam blocks, a pelvic ring injury with symphysis and disruption of SI-joint and a pelvic ring fracture with symphysis disruption and transforaminal sacral fracture. One examiner performed both visual and virtual controlled reduction. To measure the residual displacement a magnetic motion tracking device was used. The results revealed significantly increased residual displacement with virtual compared with visual control. The differences were low, averaging 1 $\mathrm{mm}$ residual translation and $0.7^{\circ}$ angulation respectively. This residual displacement may not be clinically relevant. Further development of the software prototype as integration of surface registration may lead to improved handling and facilitated multifragment tracking. Use in the clinical setting seems possible within a short time.
\end{abstract}

\section{Introduction}

Computer assisted surgery was introduced to increase the accuracy of selected procedures in orthopaedic and trauma surgery. Current clinically available software modules for CT based optoelectronic navigation systems are restricted to navigate a limited number of instruments or implants (pointer, chisels, pedicle probe, drill) within one solid bony structure. A real time visualization of fragment manipulation is not available for clinical applications, yet.

However, reduction is one of the key procedures in fracture surgery and can influence the immediate course of an operation, but the quality of reduction also directly affects the long-term outcome in the many cases. fractures This has been reported in several series especially for pelvic ring and acetabular fractures.

Within the current study we evaluated a new developed software for accuracy allowing independent registration and tracking of fragments. 


\section{Material and Methods}

For the experiments a commercially Navigation system was used (Surgigate ${ }^{\circledR}$ Medivision, Oberdorf, Switzerland). The system included an Ultra 10 workstation (SUN Microsystems, Palo Alto, CA,) and an Optotrak 3020 optoelectronic localizer (Northern Digital Inc., Waterloo, Canada).

An alpha-version software for virtual controlled reduction based on standard software (Surgigate ${ }^{\circledR}$ Medivision, Oberdorf, Switzerland) was developed in cooperation with the Maurice Müller Institute of Biomechanics in Berne, Switzerland. The reduction software was developed within two major software platforms (imaging Application Platform -Cedara, Missassuga, Canada and Open Inventor, SGI, Mountain View, CA). The combination of this software allows the system to work with volumetric datasets (Voxel graphic) extracted from conventional CT datasets and surface models (vector graphics). The automatic generation of surface models from voxel data enables real time tracking of bone fragment motion. Currently registration is limited to paired-point matching and tracking is limited to two fragments.

\subsection{Fracture Models}

Commercially available plastic models $(n=2)$ of the whole bony pelvic ring and osteoligamentous anatomic specimens $(n=2)$ were used for similar experiments. Two different injury types were created:

combined symphysis and SI-joint disruption AO/OTA61 C 1.2 [1] and combined symphysis disruption and transforaminal sacral fracture AO/OTA 61 C 1.3 [1]

From each single object $\mathrm{CT}$ data were acquired (Somatom +4 , Siemens, Erlangen, Germany) (140 kV, $171 \mathrm{~mA}$, slice thickness $2 \mathrm{~mm}$, reconstruction index $2 \mathrm{~mm}$, pitch 1 $\mathrm{mm}$ ). After preoperative segmentation and surface model generation a dynamic reference base was attached to each fragment. Registration was done paired point with four titanium fiducials close to the fracture lines.

\subsection{Measurement Devices}

The fragments were fixed to 3D-arms allowing three-dimensional manipulation and easy fixation within a certain position (Figure 1). For measurement of the accuracy of reduction an electromagnetic three-dimensional tracking system was attached to the fragments and calibrated in a secure distance to metal bodies (Polhemus, Colchester, VT). The accuracy of the system was specified and tested by calibration with $0.1 \mathrm{~mm}$ in the translational planes ( $\mathrm{x}, \mathrm{y}$ and $\mathrm{z}$ planes), and $0.1^{\circ}$ angulation deviation within the angles $\alpha, \beta$ and $\gamma$. The data processing was done with a new developed software.

For the reduction set-up the Pohlemus motion sensor was attached to each of the fragments (Figures 1, 2). The sensors were attached to the fragments by $105 \mathrm{~mm}$ plastic rods to avoid metallic artifacts affecting the electromagnetic motion tracker. The motion tracker unit was calibrated to an error less than $0.1 \mathrm{~mm}$ and $0.1^{\circ}$ angulation. A data acquisition rate of $1 \mathrm{~Hz}$ was used. 


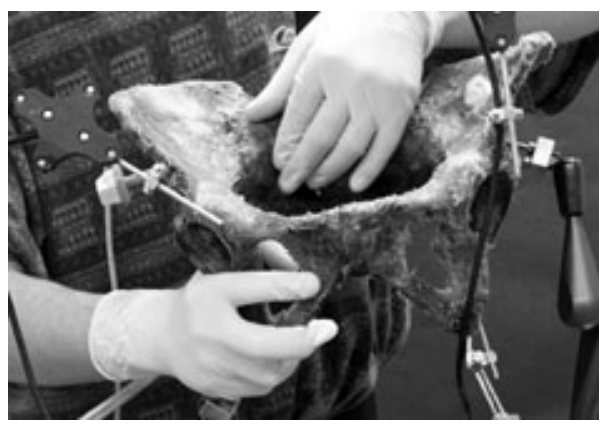

Fig. 1: For definition of the zero position the examiner reduces the fracture with full apposition without a step-off. Then, the motion tracker is calibrated to the zero position. In the same fashion visual controlled reduction takes place.

\subsection{Reduction Protocol}

First, the reduction under direct visualization and using tactile information was performed (Figure 2). Reduction was defined as complete fragment apposition without any step-off. This position was used to calibrate the motion tracker system to the start-up position with all axes and angles reset to zero.

One examiner did the reduction without limitation of time using two different methods: visual controlled reduction with full direct sight and tactile information for definition of the ideal results $(n=20)$ and solely virtual controlled reduction with the examiner completely blinded to the object only with monitor sight and manipulating only the 3D-arms $(n=20)$ (Fig. 2$)$.

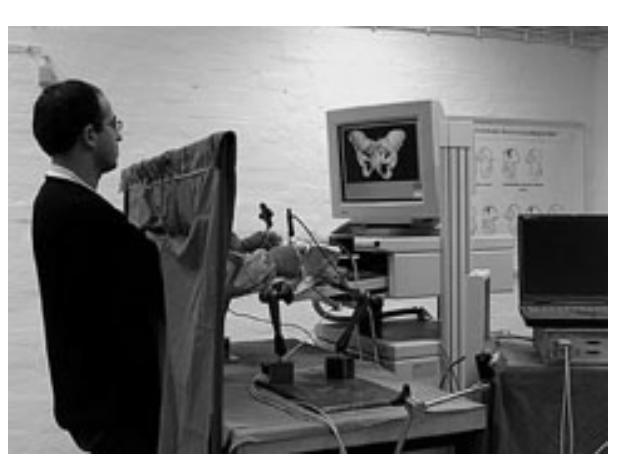

Fig. 2a: With virtual controlled reduction the examiner is blinded to the fracture model itself.

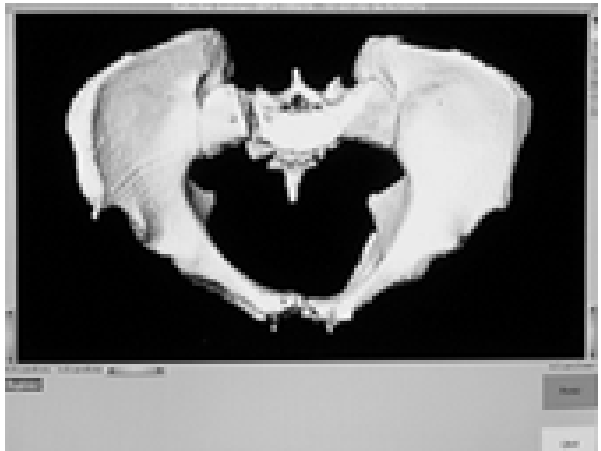

Fig. 2b: The virtual display allows all views, as an inlet view displayed here in this $\mathrm{C} 1.3$ fracture.

The experiment was started after registration when the calculated system error within the navigation system was less than $1 \mathrm{~mm}$, otherwise the registration was repeated. The endpoint of the experiment was defined as the position judged by the examiner as 
position of anatomical reduction. This was done using the view on the monitor. The $3 \mathrm{D}$-arms were then fixed rigidly preserving the end-point position. Data recording was continued for another 10 seconds in the end position to create stable end point measurements. The residual displacement was measured in the $\mathrm{x}, \mathrm{y}$ and $\mathrm{z}$ planes $(\mathrm{mm})$ and in the $\alpha, \beta$ and $\gamma$ angles (degrees). The ASCII data provided by the motion tracker system were imported to an Excel spreadsheet (Microsoft, Richmond, WA.) The resulting reduction accuracy was calculated using Euclidean geometry $d=\sqrt{ } x^{2}+y^{2}+z^{2}$. The Euclid distance (translation) and the residual angulations were calculated compared with the start-up (zero) position.

A comparison of outcomes was done using systematic error analysis between direct and virtual controlled reduction.

Statistical analysis was done using the Levene test and a nonpaired $t$ test. Significance level was set at $\mathrm{p}<0.05$. All analyses were performed using commercially available statistical software (SPSS, SPSS Inc, Chicago, IL).

\section{Results}

\section{Pelvic Models and Specimen}

C 1.2 injury. The residual translation is shown in Figures 3. Virtual controlled reduction led to increased residual translation compared with visual controlled reduction for foam models and specimen $(\mathrm{p}<0.01)$, the average maximum deviation was $0.7 \mathrm{~mm}$ for the virtual-group.

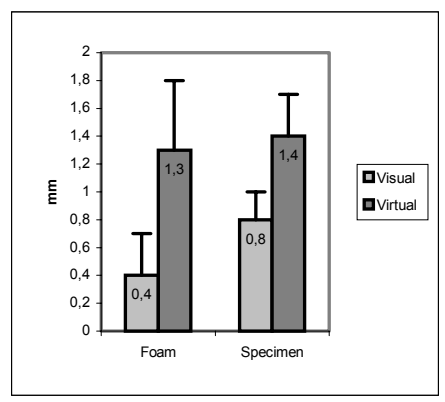

Fig. 3a: The residual translation (Euclid distance) was small after both, visual and virtual controlled reduction. With visual controlled reduction a significant lower residual translation was achieved compared to virtual control $(\mathrm{p}<0,01)$. No significant difference was appreciated between foam models and specimen $(\mathrm{p}>0,05)$.

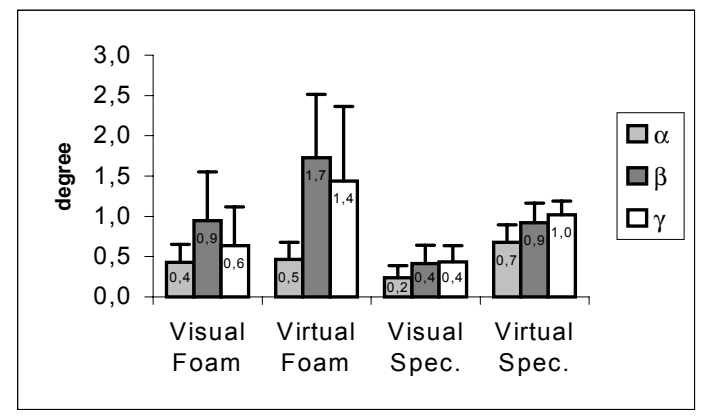

Fig. 3b: Foam models: the residual angulation was lower only for the $\square$-angle $(\mathrm{p}<0,01)$. Specimen: visual lead to lower residual angulation for all angles compared to virtual controlled reduction $(\mathrm{p}<0,05)$. The residual angulation of the specimen pelvis was lower compared to the foam pelvis models $(\mathrm{p}<0,05)$. 
After visual controlled reduction of the foam pelvis the residual angulation was less only for the and $\gamma$ angle (Figure 8). The differences averaged $0.3^{\circ}$ for the $\alpha$ angle, and $0.7^{\circ}$ for the $\beta$ and $\gamma$ angles respectively.

Compared with virtual controlled reduction visual controlled reduction with the pelvis specimen led to significantly lower residual angulation $(\mathrm{p}<0.05)$.

The residual angulation of the pelvis specimen was lower than the foam models for visual and virtual controlled reduction $(\mathrm{p}<0.05)$.

C 1.3 injury. With virtual controlled reduction residual translation was increased compared with visual control. The difference averaged $0.7 \mathrm{~mm}$. With virtual controlled reduction residual angulation for foam models was increased for the $\gamma$ angle only $(\mathrm{p}<0.05)$, whereas with the pelvis specimen virtual controlled reduction led to increased $\beta$ and $\gamma$ angles $(\mathrm{p}<0.05)$ compared with visual reduction. Generally the specimen models had lower residual angulation compared with the foam models $(\mathrm{p}<0.05)$.

\section{Discussion}

The value of currently available navigation systems has been reported in several studies. Several standard procedures, e.g. pedicle screw insertion, pelvic osteotomies and cup and shaft implantation of hip prostheses require high intraoperative precision have been included in the available software [2-7]. However, these applications are limited to one bone or one fragment. In trauma surgery $\mathrm{CT}$ based optoelectronic systems have been used for pelvic fracture surgery, including internal fixation of pelvic ring fractures or pelvic nonunion $[8,9]$. But these were situations with no further fracture movement.

Langlotz et al. introduced a new software for CAS controlled periacetabular osteotomies that allows the independent tracking of a second fragment [2]. Here the primary registration is limited to the intact pelvis and the second fragment can be generated only virtually. This software has been used successfully for osteotomies and reduction control in late reconstructions of malhealed pelvic ring fractures [10]. The software version introduced in this study overcomes these limitations by the option of independent and repetitive registration of each fragment and real time movement tracking of both. Although only paired-point registration is available, yet, it was sufficient for accurate virtual reduction control in this set-up. Furthermore the segmentation algorithm and generation of the surface models seemed reliable for foam models and specimens.

In some studies, accuracy analyses of CAS systems are related to the examination of the imaging technique [11], the camera unit [12, 13], the registration algorithms [14, 15] or to overall clinical applications. [2, 7, 16-19].

However, for the surgeon the overall clinically relevant error, as defined by Maciunas et al. [13] is a more relevant factor. This comprises the errors associated with imaging, registration and the technical accuracy of the CAS system. A standard acceptable error cannot be provided, because this is dependent on the application and the primary diagnosis. 
The current set-up focused on the clinically relevant issue of residual displacement after the process of pelvic ring reduction being monitored and controlled either by the sight and tactile information or visual control. The use of an high precision threedimensional magnetic based motion tracking device, the set-up, and the repetition of the experiments was facilitated and three-dimensional datasets were available.

The results showed differences between visual and virtual control of reduction, however, both groups are within a range which is accepted as an anamic result when applied clinically to the evaluation of pelvic reduction.

The missing soft tissue envelope which influences the quality of a closed reduction is a disadvantage of the model used in the current study. Additional experiments are planned with a more realistic set-up. The lower residual angulations seen in the specimen compared with foam models seem to be attributable to a better fit of the fragments after reduction was done.

This new developed software module allows simultaneous, independent registration of two fragments and real time representation of both fragments. This led to reproducible high precision when used for virtually controlled reduction in this experimental set-up. Integration of surface registration and enhanced surface model generation as further developments may lead to improved handling and multifragment tracking. Use in the clinic seems possible within a short time.

\section{References}

1. Orthopedic Trauma Association Committee for coding and classification, Fracture and dislocation compendium. J Orthop Trauma, 1996. 10(Suppl 1): p. V-IX.

2. Langlotz F, et al., The first twelve cases of computer assisted periacetabular osteotomy. Comput Aided Surg, 1997. 2: p. 317-326.

3. Langlotz F, et al., Computer Assistance for Pelvic Osteotomies. Clin Orthop, 1998. 354: p. 92-102.

4. Langlotz $\mathrm{U}$, et al. A novel system for complete THR planning and intraoperative freehand navigation. in CAOS Computer Assisted Orthopaedic Surgery. 2000. Davos, Switzerland.

5. Lavallee, S., et al., Computer-assisted spine surgery: a technique for accurate transpedicular screw fixation using CT data and a 3-D optical localizer. J Image Guid Surg, 1995. 1(1): p. 65-73.

6. Lavallée S, et al., Computer-assisted Spine Surgery Using Anatomy-based Registration., in Computer-Integrated Surgery, Taylor R, et al., Editors. 1996, MIT Press: Cambridge.

7. Laine $\mathrm{T}$, et al. Accuracy of pedicle srew insertion with and without computer assistance - A randomized controlled clinical study in 100 consecutive patients. in CAOS Computer Assisted Orthopaedic Surgery. 2000. Davos, Switzerland.

8. Kahler DM, Zura RD, and Mallik K. Computer guided placement of iliosacral screws compared to standard fluoroscopic technique. in 5th Symposium on CAOS Computer Assisted Orthopaedic Surgery. 2000. Davos.

9. Zura, R.D. and D.M. Kahler, A transverse acetabular nonunion treated with computer-assisted percutaneous internal fixation. A case report. J Bone Joint Surg Am, 2000. 82(2): p. 219-24.

10. Hüfner T, et al. Computer assisted surgery for correction of a malhealed pelvic ring fracture in a young female patient. in 4th CAOS Computer Assisted Orthopedic Surgery. 1999. Davos. 
11. Schneider, J., Risikomanagement in der Medizintechnik. Methodik zur Überprüfung der geometrischen genauigkeit CT-basierter Verfahren in der computer-unterstützten Therapie., in Medizinische Fakultät. 2000, Friedrich-Alexander-Universität: Erlangen-Nürnberg. p. 87.

12. Chassat F and Lavallee S. Experimental protocol of accuracy evaluation of 6-D localizers for computer-integrated surgery: application to four optical localizers. in Proceedings of the First International Conference on Medical image computing and computer-assisted intervention - MICCAI '98. 1998. Berlin Heidelberg: Springer.

13. Maciunas, R.J., R.L. Galloway, Jr., and J.W. Latimer, The application accuracy of stereotactic frames. Neurosurgery, 1994. 35(4): p. 682-94; discussion 694-5.

14. Bächler R, Oberflächenbasierte Registrierung für orthopädische und HNOAnwendungen., in Philosophisch-naturwissenschaftliche Fakultät. 2000, Universität Bern: Bern, Switzerland. p. 114.

15. Hüfner T, et al. Registration using a modified external fixateur system for the pelvis. in 5th International Symposium on CAOS. 2000. Davos.

16. Laine, T., et al., Accuracy of pedicle screw insertion: a prospective CT study in 30 low back patients. Eur Spine J, 1997. 6(6): p. 402-5.

17. Nolte LP, et al., Image-guided insertion of transpedicular screws. Spine, 1995. 20(4): p. $497-500$.

18. Merloz, P., et al., Pedicle screw placement using image guided techniques. Clin Orthop, 1998(354): p. 39-48.

19. Vandervelde D, Mahieu G, and Nuyts R. Reduction in the variability of acetabular cup positioning using computer assisted surgery. in CAOS Computer Assisted Orthopaedic Surgery. 2000. Davos. 